\title{
PERSONALIZED WEB SEARCH USING KEY PHRASE-BASED USER PROFILES
}

\author{
Sara Abri ${ }^{1}$, Rayan $\mathrm{Abri}^{1}$ and Salih Cetin ${ }^{2}$ \\ ${ }^{1}$ Department of Computer Engineering, Hacettepe University, Ankara, Turkey \\ ${ }^{2}$ Mavinci Informatics Inc., Ankara, Turkey
}

\begin{abstract}
In the context of the personalized web, the structure of user profiles remains a challenge due to its effect on information retrieval efficiency. In creating a user profile, how to extract collections of metadata of documents is a significant point. Although the efficiency of the structures such as keyword-based has proven, there is not research on key phrase based user profiles in the process of personalization. Previous methods to create user profiles emphasize keyword extraction while ignoring the existence of key phrases in the documents. This article proposes the use of keyphrase-based user profiles in personalized web search. We investigate the state of the art keyphrase extraction algorithms by considering different models of supervised and unsupervised methods. This can overcome the problems of missing keyphrases in user profiles and increase the accuracy of information retrieval. Finally, we evaluate keyphrase-based user profiles using a re-ranking algorithm to complete the process of personalization using different datasets. Personalized models based on supervised keyphrase extraction approaches obtained more accuracy by $7 \%$ than unsupervised approaches.
\end{abstract}

\section{KEYWORDS}

Personalized Web Search, Keyphrase Extraction, Graph-based Methods, User Profiling

\section{INTRODUCTION}

In the personalization process, user profiles are created using user-specific content, user behavior, and user context. The structure of the user profile is an important issue because of its impact on ranking performance. It is clear that if the structure used in the user profile is more robust, the user profile and personalized services result in better efficiency. Therefore, the process of generating user profiles is a difficult problem, and constructing accurate and efficient user profiles is still an open challenge in personalized information retrieval.

A keyphrase consists of a set of word expressions. This set of words can be an indicator of the content or hidden topics of a document. Extracted keyphrase can be used in the fields such as information retrieval (Medelyan, O. et al, (2009)), summarization (Dredze, M. et al, (2008)), clustering (Hammouda, K.M. et al, (2005)), categorization (Hulth, A. and Megyesi, B.B. (2006)) and indexing (Gutwin, C. et al. (1999)) in the text. In this paper, several supervised and unsupervised methods have been proposed to extract keyphrases. The unsupervised methods consist of statistical approaches, topic-based clustering groups, and graphed-based methods. We evaluate several graph-based methods since those are the most commonly used. Among supervised methods, we implemented Kea and WINGNUS to compare with unsupervised methods.

We also conduct comparative research on keyphrase-based user profiles to consider keyphrase-based user profiles in the personalization process. To evaluate the method in the process of personalization, we use ranking approaches for re-ranking the result of respect to the user profiling and the submitted query by the user. The organization of the paper is as follows. Section 2 discusses the related work on keyphrase extraction and personalization. The method including creating keyphrase based user-profiles and the re-ranking process is presented in Section 3 in detail. Evaluation methodology and results are presented in Section 4. Section 5 concludes the discussion. 


\section{RELATED WORK}

\subsection{Personalization}

There are different ways to create user profiles such as keyword-based or term-based (Matthijs, N. and Radlinski, F. (2011), Daoud, M. et al. (2009)), topic-based (Abri, S and Abri, R.(2020), Harvey, M. et al, (2013) and Abri, S. et al. (2020)) and ontology-based (Sieg, A. et al. (2007), Chirita, A. et al. (2005)) however the main focus to create user model is on the keyword-based in the personalized process. For example, in the personalization process Liu, F. et al. (2004), Karimi, S. and Abri, R. (2016) have created keyword-based profiles by the user's search histories to improve retrieval effectiveness in personalized web search. Weighted keyword vectors as a predominant representation of user preferences and interests as hierarchical concepts in some cases have increased (Santos, R. L. et al. (2010)). Chirita, A. et al. (2005) used a classification on ODP metadata to personalize search and Qiu, G. et al. (2007) used ODP for classifying topics and explored their relations with query ambiguity. Vicente-López, E. et al. (2014) conducted a study on different user profile representation approaches, and Amer, N. et al. (2016) investigated word embedding in the field of personalization.

To employ a keyword-based model for web personalization, it is needed to acquire keywords from documents visited by the users the same as Barla, M. and Bieliková, M. (2010). They have developed the JKeyExtractor library to extract keywords from web pages to make user models.

There are several modified versions of keyword-based user models. For example, Wei, C. et al. (2009) tried to solve a lack of semantic information of keywords and designed a user profile based on the category knowledge base. In this paper, the author attempts to improve the user model by combining the keywords and ontology concepts. Since user interests and search intentions are changing during a search session, long term and short term profiles were also discussed (Vu, T. et al. (2017) and Bennett, P. et al. (2012)). Vu, T. et al. (2017) created temporal user profiles using the user's clicked documents and the profile was used for the ranking process. In another research $\mathrm{Vu}$, T. et al. (2015) also used three typical time scales for building a long-term profile, a daily profile, and a session profile, and the results were improved using click entropy and query position in a search session.

\subsection{Keyphrase Extraction}

Keyphrase extraction methods are divided into supervised and unsupervised. Various unsupervised methods consist of statistical approaches, topic-based clustering groups, and graphed-based methods. In unsupervised methods, it is used several graph-based methods since those are the most commonly used. From supervised methods, we implemented Kea and WINGNUS to compare with unsupervised methods. Unsupervised methods consist of corpus-dependent and corpus-independent. Most of the corpus-independent methods are graph-based.

Graph-based keyphrase extraction introduced with TextRank by Mihalcea and Tarau (2004) is applied to rank words based on their associations in the graph. Keyphrases are formed by adjacent keywords in the document. The edges may be weighted, like in SingleRank (Xiaojun, W. and Jianguo, X. (2008)), using the number of co-occurrences as weights. The nodes are scored using some node ranking metric, such as degree centrality or PageRank. Chris, M. et al. (2015) used a WordAttractionRank approach to incorporate the distance between the words.

Keywords and keyphrases can be used to enrich the presentation of search results and help users to understand the main topics of a Web page. Using keywords in creating user profiles is explored in various research however there is a little research on investigating the state of the art methods in keyphrase extraction to create keyphrase based user profiles in the process of personalization. For example, Hulth (2004) described a system along the keywords extraction process for web pages returned by Google search engine. Therefore we decided to investigate how personalization can be integrated into the state of the art keyphrase extraction models. 


\section{USER PROFILING PROCESS}

The user modeling process is to capture, record, and manage user needs and interests, by creating a user profile. The system developed by Tang, J. et al. (2010) constructed user profiling into three sub-tasks consisting of profile extraction, integration, and interest discovery. A simple user profile is based on terms of all relevant feedback containing stemmed terms, frequency, and weight. A user profiling process can be conducted into the following subprocess, collecting information like behaviors, pre-processing of collected information, and analyzing information to create a user model. Finally, technology or application exploits the created user profile to provide personalized services.

\subsection{Collecting and Pre-Processing User Data}

Older systems gathered data explicitly from the users while recent research is focused on implicit methods or behavioral user profiling for collection information. There are two major matters of collecting information. The content of the data collected and techniques to collect information. The content of the data includes browsing data, bookmarking data, click-through data while methods to collect data are divided into explicit and implicit methods. In more research, implicit methods are preferred because of the access to large sources of data in contrast to explicit methods with a few data. Using an implicit dataset we can study many different users interactions while it will be infeasible with explicit data. In this paper, we use clicked pages by a user to create a user profile.

After the collection of relevant information, it might need a preprocessing process to remove duplicate and clean up the data. Besides, the extracted URL or document also needs to be cleaned. To clean, we extracted the content of clicked pages by users and then did a pre-processing on extracted documents. To do this, we did a collection of word tokenization, sentence segmentation, steaming, stop-word removing, and parsing process provided by the python Natural Language Toolkit (Nltk) for English. The candidate keyphrases are stemmed using porter steaming on words to reduce the number of mismatches.

\subsection{Personalization Using Keyphrase Based Profiles}

We create user profiles in the form of keyphrases extracted of clicked documents by users using keyphrase extraction methods. We use a number of models such as TF-IDF, RAKE (Stuart, R., et al. (2010)), TopicRank (Bougouin, A. et al. (2013)), TextRank (Mihalcea and Tarau (2004)), Kea(Keyphrase Extraction Algorithm) Witten, IH. et al. (1999) and WINGNUS (Thuy, D. et al. (2010)) to extract keyphrases. Current methods in unsupervised are divided into statistical models and graph-based models. Among statistical models, TF-IDF is used successfully and we intended to use it as a basic algorithm to the comparison. TF-IDF described in Equation 1. can be used for stop-words filtering in various subject fields including text summarization and classification.

$$
w_{i, j}=t f_{i, j} \times \log \frac{N}{d f_{i}}
$$

Where $t f \quad{ }_{i j}$ and $d f_{i}$ are the number of occurrences of $i$ in $j$ and the number of documents containing $i$. $N$ is the total number of documents. In keyphrases extraction algorithms, in the first step, keyphrase candidates are extracted from the content of the document, then candidates are ranked using a weighting mechanism or a machine learning technique as unsupervised or supervised. Finally, the top-K highest weighted candidates are selected. More on unsupervised keyphrase extraction methods, we apply efficient graph-based models such as RAKE, TopicRank, and TextRank. In graph-based models, a graph is built based on words or phrases where the edges' weights are computed using co-occurrence counts (Xiaojun, W. and Jianguo, X. (2008) and Yutaka, M., and Mitsuru, I. (2004)).

The RAKE algorithm proposed by Stuart, R., et al. (2010), is a language-independent method that extracts keywords by analyzing word frequency and its co-occurrence with other words in the text. RAKE focuses on finding multi-word phrases containing frequent words. First, RAKE splits the text into sentences using punctuation signs and generates the candidates. All words listed in the stop-word file will be treated as phrase boundaries. RAKE is based on the theory that keyphrases frequently contain multiple words but rarely contain 
standard punctuation or stop words or other words with minimal lexical meaning, however, it won't work in cases where the stop-word is part of the phrase.

The TextRank algorithm proposed by Mihalcea and Tarau (2004), is an algorithm based on PageRank and the same as RAKE extracts key phrases by a co-occurrence graph. In this algorithm, documents are split into the sentences, and the words with specific tags such as noun, prop, and verb. Then a graph of words is created and the weight for each node is calculated. It collects the influence of each of its connections and determines the new score for the node. In this way, TextRank considers the similarity between each sentence to all other sentences.

The TopicRank algorithm proposed by Bougouin, A. et al. (2013), is an improvement of the TextRank that extracts key phrases using a topical representation of the document. Candidate keyphrases are clustered into topics and used as vertices in a graph. A graph-based ranking model is applied to assign a score to each topic. In the TopicRank approach, ranking topics instead of words is a more straightforward way to identify the set of keyphrases that covers the main topics of a document. Also, the use of a complete graph captures the semantic relations between topics.

Given a complete graph as $\mathrm{E}=\left\{\left(v_{1}, v_{2}\right) \mid \forall v_{1}, v_{2} \in \mathrm{V}\right\}, v_{1} \neq v_{2}$ of $\mathrm{V} \times \mathrm{V}$, Vertices are topics and the edge between two topics $t_{i}$ and $t_{j}$ is weighted according to the strength of their semantic relation. $t_{i}$ and $t_{j}$ have a strong semantic relation if their keyphrase candidates often appear close to each other in the document. Therefore, the weight $w_{i, j}$ of their edge is defined as defined equations by Bougouin, A. et al. (2013):

$$
\begin{aligned}
w_{i, j} & =\sum_{c_{i} \in t_{i}} \sum_{c_{j} \in t_{j}} \operatorname{dist}\left(c_{i}, c_{j}\right) \\
\operatorname{dist}\left(c_{i}, c_{j}\right) & =\sum_{p_{i} \in \operatorname{pos}\left(c_{i}\right)} \sum_{p_{j} \in \operatorname{pos}\left(c_{j}\right)} \frac{1}{\left|p_{i}-p_{j}\right|}
\end{aligned}
$$

Where $\operatorname{dist}\left(c_{i}, c_{j}\right)$ is the reciprocal distance between the offset positions of the candidate keyphrases $c_{i}$ and $c_{j}$ and $\operatorname{pos}_{i}$ represents all the offset positions of the candidate keyphrase $c_{i}$. After creating a graph, the ranking model TextRank, is used to rank the topics. This ranking model considers a score to topics based on the concept of voting that high scoring topics contribute more to the score of their connected topic $t_{i}$.

$$
S\left(t_{i}\right)=(1-\lambda)+\lambda \times \sum_{t_{j} \in V_{i}} \frac{w_{i, j} \times S\left(t_{j}\right)}{\sum_{t_{k} \in V_{j}} w_{j, k}}
$$

Where $v_{i}$ are the topics voting for $t_{i}$ and $\lambda$ is a damping factor generally defined to 0.85 . In the following to complete the benchmark, we evaluate supervised models like Kea and WINGNUS as baselines methods. Kea builds a classifier based on the Bayes Theorem using training documents, and it uses the classifier to extract keyphrases from new documents. In the training and extraction phases, KEA analyzes the input document depending on orthographic boundaries (such as punctuation marks, newlines, etc.) and exploits two features: TF-IDF and the first occurrence of the term. In WINGNUS keyphrase candidates are simplex nouns and noun phrases detected using a set of POS filtering rules. Keyphrases are then selected using a Naive Bayes classifier with a large set of features including document logical structure information. Finally extracted keyphrases are stored in the keyphrase based user profiles in the form of XML and in the next step to be applied in the ranking process.

Re-ranking documents for a new query using a keyphrase based user profile, we measure the similarity between the extracted keyphrases of each document in the query log and the keyphrase based user profile for each user by cosine similarity. The cosine similarity is useful because of measures changing between similar documents regardless of the size of the document. It can be measured using Equation 5.

$$
\cos (\theta)=\frac{\vec{a} \cdot \vec{b}}{\|\vec{a}\|\|\vec{b}\|}=\frac{\Sigma a_{i} b_{i}}{\sqrt{\Sigma a_{i}^{2}} \sqrt{\Sigma b_{i}^{2}}}
$$

Where $\vec{a} . \vec{b}=\Sigma a_{i} \cdot b_{i}=a_{1} b_{1}+a_{2} b_{2}+\ldots+a_{n} b_{n}$ is the dot product of the two vectors. For the evaluation re-ranking process using keyphrase based user profile, we tried a re-ranking algorithm on the data set and the results are presented in Chapter 4. Besides, we need to consider time in creating user profiles. To build short-term and long-term user profiles, we consider user profiles for one and three months as $\mathrm{Vu}$, $\mathrm{T}$. et al. (2015) and Momtazi, S., and Lindenberg, F. (2016). 


\section{EVALUATION}

\subsection{Preparing and Cleaning Data Set}

In this paper, evaluation is made on two datasets to investigate the precision of methodology. In the first experiment, we used the AOL data set including the query log collected from 650k users. To clean the data, we first selected those queries which resulted in a click on a URL. In the second experiment, to achieve a more dominant result, we used the data set provided by TREC 2014 Session Track. The Session Track consists of 1021 query sessions for 60 different topics along with the clicked result and user id.

\subsection{Evaluation Parameters}

To evaluate the personalized model, we divided the dataset into $95 \%$ for training and the last $5 \%$ of queries for testing. The performances of personalization are evaluated in terms of success at rank $\mathrm{k}(\mathrm{S} @ \mathrm{k})$, the mean reciprocal rank (MRR), and discounted cumulative gain (DCG) up to rank 10 when a maximum of 10 key phrases are extracted $(\mathrm{k}=10)$ to create user profiles. To compare with a baseline method, we use a non-personalized model as a generic ranking without personalization created based on Latent Dirichlet Allocation (LDA) topic model. Building on the same framework introduced by Harvey, M. et al, (2013) and the results presented by Abri S. et al. (2020), documents are ranked with respect to the LDA model $P(d \mid q)$ called NonPTM (Non-Personalized Topic Model) here.

$$
\begin{aligned}
\operatorname{NonPTM}(d, q) & =P(d \mid q) \propto P(d) P(q \mid d) \\
& =P(d) \prod_{w \in q} P(w \mid d) \\
& =P(d) \prod_{w \in q} \sum_{z} P(w \mid z) P(z \mid d)
\end{aligned}
$$

Where $P(d)$ is the prior document probability and $z$ is the topic latent variable estimated using LDA. $P(w \mid z)$ and $P(z \mid d)$ are obtained from the LDA topic model. The number of topics used for LDA is an important parameter. Figure 1. shows the MRR for different topic numbers ranging from 10 topics to 100 for two datasets. The results indicate that using 40 topics yields the best results in the AOL dataset and 30 topics for the TREC2014 dataset.

We compare the performance of the proposed method with the state-of-the-art algorithms using an open-source python-based keyphrase extraction toolkit, called pke. The pke toolkit is open source and available at https://github.com/boudinfl/pke. To investigate the effect of various parameters in rake, we explored these parameters depending on the text. It has shown in Figure 2. the parameters that performed best on the current dataset with at most three keywords in each phrase.

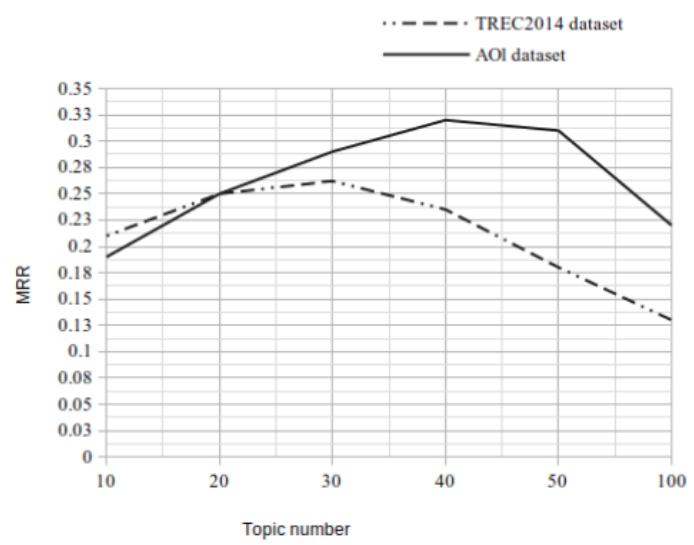

Figure 1. The Changes in MRR with different topic numbers in LDA model

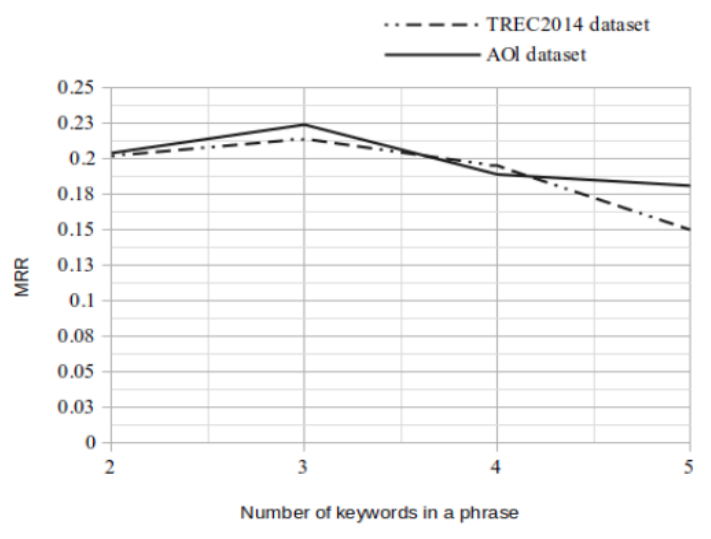

Figure 2. The MRR changes in phrase length in Rake 


\subsection{Experimental Result}

To evaluate the proposed model we provided two experiments with different datasets. In the first experiment, we used the AOL query log data set as a big resource of explicit query log data. In both of the data sets, clicked documents are a reference in building user profiles. We will present the result of the experiments in the following. The models are called as personalized keyphrase extraction(PKM). For example, PKM-TextRank is a personalized ranking model based on TextRank keyphrase extraction. In this model, TextRank keyphrase extraction is used in creating user profiles.

Tables 1. and 2. report the MRR, S@1, S@10, and nDCG@10 scores for Non-persobnalized topic model(Non-PTM) and personalized keyphrase extraction(PKM) models in AOL and Session Track 2014 datasets. In Table 1., the columns represent the used methods including Non-PTM, TextRank, Rake, and TF-IDF. To consider the time dimension in the process of user profiling, we separated profiles as short-term and long-term user profiles. As depicted in Table 1., the best results are obtained with the Non-PTM and TextRank methods. In Personalization using the TextRank(PKM-TextRank) model, the MRR is $32 \%$ for short-term and $26 \%$ for the long-term while it needs more time to run rather than Rake and TF-IDF. It can be seen using the results that in all methods created based on the short-term profiles are more highly efficient than the long-term profiles.

Table 1. Ranking Performance of Non-PTM and personalized keyphrase based models on the AOL data set

\begin{tabular}{l|ccc|ccc|ccc|ccc} 
& \multicolumn{3}{|c|}{ Non-PTM } & \multicolumn{3}{|c|}{ PKM-TextRank } & \multicolumn{3}{c|}{ PKM-Rake } & \multicolumn{3}{c}{ PKM-Tfidf } \\
& S@1 & S@10 & MRR@10 & S@ & S@10 & MRR@10 & S@1 & S@10 & MRR@10 & S@1 & S@10 & MRR@10 \\
\hline Long-Term & 0.205 & 0.371 & 0.267 & 0.201 & 0.353 & 0.259 & 0.181 & 0.310 & 0.224 & 0.172 & 0.308 & 0.218 \\
Short-Term & 0.282 & 0.436 & 0.330 & 0.280 & 0.410 & 0.325 & 0.250 & 0.368 & 0.297 & 0.255 & 0.358 & 0.296 \\
\hline
\end{tabular}

Table 2 represents the ranking score when using non-personalization and personalization based on keyphrase based profiles using a set of supervised and unsupervised approaches. Among supervised approaches, Kea and WINGNUS are used as basic approaches while the best results (\%35 in nDCG@10)are obtained using created models using these methods. In unsupervised methods also we tried TextRank, Rake, and TopicRank as graph-based methods and TF-IDF as a feature-based unsupervised method. As shown in Table 2., PKM-TF-IDF resulted in the best performance in nDCG@10 among all approaches. To investigate the effect of the time factor in creating a user profile on the SessionTrack dataset, we compared session-based and long-term based profiles. The result is depicted in Figure 3. As the result shows supervised approaches (Kea and WINGNUS) have provided more successful results although those are needed for training data and so the bias towards the domain on which they are trained is a critical issue. The tendency in performance in session-based profiles is more than long-term profiles in all personalized models.

Table 2. Ranking Performance of Non-PTM and personalized keyphrase based models on the Session Track 2014

\begin{tabular}{c|cccc} 
& S@1 & S@10 & MRR@10 & DCG@10 \\
\hline Nonpersonalized topic-based model(Non-PTM) & 0.216 & 0.340 & 0.262 & 0.291 \\
\hline Personalized keyphrase-based models(PKM) & & & & \\
PKM-TextRank & 0.197 & 0.318 & 0.238 & 0.263 \\
PKM-Rake & 0.175 & 0.303 & 0.214 & 0.249 \\
PKM-TopicRank & 0.204 & 0.326 & 0.240 & 0.272 \\
PKM-Tfidf & 0.213 & 0.335 & 0.257 & 0.280 \\
\hline PKM-Kea & 0.257 & 0.390 & 0.304 & 0.348 \\
PKM-WINGNUS & 0.260 & 0.395 & 0.310 & 0.352
\end{tabular}




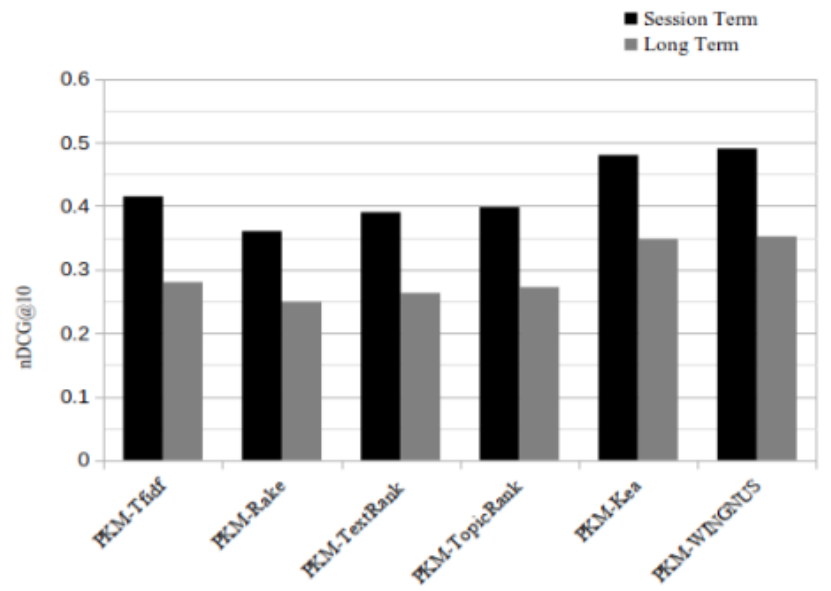

Figure 3. Ranking performance of the six models PKM-TF-IDF, Rake, TextRank, Kea,WINGNUS and TopicRank using nDCG metric

\section{CONCLUSION}

In this paper, keyphrase based user profiles in the personalized web search are investigated. In this way, it is considered how integration between keyphrase extraction and personalization by state-of-the-art approaches. The personalization models are created using the state of the art keyphrase extraction approaches considering supervised and unsupervised methods. For evaluation of the model, keyphrase based user profiles using a re-ranking algorithm are applied using different datasets. The personalized models based on the supervised keyphrase extraction approaches obtained more accuracy around 7\% than unsupervised approaches. In our experiments, among supervised approaches, both feature-based method and graph-based methods resulted in the same improvement average by $26 \%$ in normalized DCG score for long-term compared to $36 \%$ for session-term profiles created using Session Track2014 dataset.

\section{REFERENCES}

Abri, S and Abri, R. 2020. Providing a personalization model based on fuzzy topic modeling. Arabian Journal for Science and Engineering.

Abri, S. et al. 2020. Group-based Personalization Using Topical User Profile. UMAP '20 Adjunct Proceedings of the 28th ACM Conference on User Modeling, Adaptation and Personalization. July 2020, pp 181-186.

Abri S. et al. 2020. A Classification on Different Aspects of User Modelling in Personalized Web Search, 4th International Conference on Natural Language Processing and Information Retrieval.

Abri S. et al. 2020. Estimating Personalization Using Topical User Profile, In 12th International Conference on Knowledge Discovery and Information Retrieval.

Amer, N. et al. 2016. Toward word embedding for personalized information retrieval. Neu-IR: The SIGIR 2016 Workshop on Neural Information Retrieval.

Barla, M. and Bieliková, M. 2010. Ordinary Web Pages as a Source for Metadata Acquisition for Open Corpus User Modeling. In: Proceedings of the IADIS International Conference WWW/Internet 2010, pp. 227-233.

Bennett, P. et al. 2012. Modeling the impact of short- and long-term behavior on search personalization. In: Proceedings of the 35th international ACM SIGIR conference on Research and development in information retrieval, pp. 185-194.

Bougouin, A. et al. 2013. Topicrank: Graph-based topic ranking for keyphrase extraction. In Proceedings of IJCNLP, pp. 543-551.

Chirita, A. et al. 2005. Using ODP metadata to personalize search. In: Proceedings of the 28th annual international ACM SIGIR conference on Research and development in information retrieval, pp 178-185. 
Chris, M. et al. 2015. Corpus- independent Generic Keyphrase Extraction Using Word Embedding Vectors.

Daoud, M. et al. 2009. A session based personalized search using an ontological user profile. In: Proceedings of the 2009 ACM symposium on Applied Computing, pp. 1732-1736.

Dredze, M. et al, 2008. Generating summary keywords for emails using topics. In: Proceedings of the 13th International Conference on Intelligent User Interfaces, pp. 199-206.

Gutwin, C. et al. 1999. Improving browsing in digital libraries with keyphrase indexes. Decision Support Systems, Vol. 27, No. (1-2), pp 81-104.

Hammouda, K.M. et al, 2005. CorePhrase: Keyphrase extraction for document clustering. In: Proceedings of the 4th International Conference on Machine Learning and Data Mining in Pattern Recognition, pp. 265-274.

Harvey, M. et al, 2013. Building user profiles from topic models for personalised search. In: Proceedings of the 22nd ACM international conference on Conference on information \& knowledge management, $\mathrm{pp}$. 2309-2314.

Hulth, A., 2004. Combining machine learning and natural language processing for automatic keyword extraction. Stockholm University.

Hulth, A. and Megyesi, B.B. 2006. A study on automatically extracted keywords in text categorization. In: Proceedings of the 21st International Conference on Computational Linguistics and the 44th Annual Meeting of the Association for Computational Linguistics, pp. 537-544.

Karimi, S. and Abri, R. 2016. Improvement of Semantic Search Results with Providing an Updatable Dynamic User Model, International Journal of Computer Applications(IJCA), Vol. 155 , No. 4,pp. 7-14.

Liu, F. et al. 2004. Personalized web search for improving retrieval effectiveness. IEEE Transactions on Knowledge and Data Engineering, Vol. 16, No. 1, pp. 28-40.

Matthijs, N. and Radlinski, F. 2011. Personalizing web search using long term browsing history. In: Proceedings of the fourth ACM international conference on Web search and data mining, pp 25-34.

Medelyan, O. et al, 2009. Human-competitive tagging using automatic keyphrase extraction. In: Proceedings of the 2009 Conference on Empirical Methods in Natural Language Processing, pp. 1318-1327.

Mihalcea, R. and Tarau P., 2004. Textrank: Bringing order into texts. In Proceedings of EMNLP 2004, pp. 404-411.

Momtazi, S. and Lindenberg, F. 2016. Generating query suggestions by exploiting latent semantics in query logs. Journal of Information Science, pp 437-448.

Qiu, G. et al. 2007. Quantify query ambiguity using odp metadata. In: Proceedings of the SIGIR '07, pp. 697-698.

Santos, R. L. et al. 2010. Selectively diversifying web search results. In: Proceedings of the CIKM '10, pp. 1179-1188.

Sieg, A. et al. 2007. Web search personalization with ontological user profiles. In: Proceedings of the sixteenth ACM conference on Conference on information and knowledge management, pp 525-534.

Stuart, R., et al. 2010. Automatic keyword extraction from individual documents. Text Mining: Applications and Theory, pp. 1-20.

Tang, J. et al. 2010. A Combination Approach to Web User Profiling. In: ACM Transactions on Knowledge Discovery from Data, Vol. 5, No. 1

Thuy, D. et al. 2010. Wingnus: Keyphrase extraction utilizing document logical structure. In Proceedings of SemEval, pp. 166-169.

Vicente-López, E. et al. 2014. Personalization of Parliamentary Document Retrieval using different User Profiles. In: Proceedings of the 2nd International Workshop on Personalization in eGovernment Services and Applications.

$\mathrm{Vu}, \mathrm{T}$. et al. 2015. Modelling time-aware search tasks for search personalisation. In: Proceedings of the 24th International Conference on World Wide Web, pp. 131-132.

$\mathrm{Vu}, \mathrm{T}$. et al. 2015. Temporal latent topic user profiles for search personalisation. In: ECIR:37th European Conference on IR Research, pp. 605-616.

$\mathrm{Vu}$, T. et al. 2017. Personalised Query Suggestion for Intranet Search with Temporal User Profiling. In: Proceedings of the 2017 Conference on Conference Human Information Interaction and Retrieval, pp. 265-268.

Wei, C. et al. 2009. A Personalized Model for Ontology-driven User Profiles Mining. 2009 International Symposium on Intelligent Ubiquitous Computing and Education, pp. 484-487.

Witten, IH. et al. 1999. Kea: practical automatic keyphrase extraction. In: ACM conference on digital libraries. New York, NY, USA, pp. 254-255.

Xiaojun, W. and Jianguo, X. 2008. Single Document Keyphrase Extraction Using Neighborhood Knowledge. In AAAI, Vol. 8. pp 855-860.

Yutaka, M. and Mitsuru, I. 2004. Keyword extraction from a single document using word co-occurrence statistical information. International Journal on Artificial Intelligence Tools, vol. 13, No. 1, pp. 157-169. 